Physica B 175 (1991) 226--230

North-Holland

\title{
Coulomb-blockade oscillations in a quantum dot
}

\author{
A.A.M. Staring, J.G. Williamson, H. van Houten and C.W.J. Beenakker \\ Phulp, Research Laboratortes, 5600 JA Endhoven. The Netherlands \\ L.P. Kouwenhoven \\ Delft Unversuv of Technology, Faculty of Physus, 2600) GA Delft, The Netherlands \\ C.T. Foxon \\ Phllp's Research Laboratones, Redlull RHI 5HA, UK
}

\begin{abstract}
Periodic oscillations as a function of gate voltage have been observed in the conductance of a quantum dot defined in a two-dimensional election gas by thee pars of gates They aic interpreted as Coulomb-blockade oscillations The amplitude of the oscillations can be enhanced up to $e^{2 / h}$ in the quantum Hall effect regime
\end{abstract}

The advent of epitaxial crystal-growth techniques, which offer control down to the scale of single atomic layers, has created new semiconductor materials of extreme purity. Advances in nano-lithography allow these materials to be structured into systems showing a variety of new phenomena. An example is the quantized conductance of a quantum point-contact $[1,2]$, which is a constriction in the two-dimensional electron gas (2DEG) in a GaAs-AlGaAs heterostructure of width comparable to the Fermi wavelength. The conductance quantization has a direct analogy in the discretized optical transmission cross-section of an aperture or slit [3].

Point contacts can be used in a variety of ways [4]. In this paper we consider their use as tunnel barriers for the study of transport through a quantum dot which is weakly coupled to two clectron reservoirs [5-9]. At temperatures $k_{\mathrm{B}} T \leqslant \Delta E$, where $\Delta E$ is the average energy-level separation in the quantum dot, transport of noninteracting electrons occurs by resonant tunneling. This is analogous to the resonant transmission of light through a Fabry-Perot interferometer. If the capacitance $C$ of the quantum dot is small, however, the electrostatic energy $e^{2} / 2 C$ required to add a single elementary charge to the quantum dot can exceed the thermal energy $k_{\mathrm{B}} T$, at low temperatures. Tunneling then is inhibited. This is known as the Coulomb blockade of tunneling, which has been studied extensively in metallic tunnel junctions [10]. Due to the large density of states, the discreteness of the energy spectrum can be neglected in such systems. This is not the case for semiconductor nanostructures, which allow a study of single electron tunneling in the quantum regime $k_{\mathrm{B}} T \leqslant$ $\Delta E$ [11]. In addition, the quantum Hall effect regime is accessible in these systems.

A manifestation of the Coulomb blockade is the appearance of oscillations in the conductance as a function of gate voltage, at temperatures above those expected for resonant tunneling of non-interacting electrons. Such "Coulomb-blockade oscillations" in semiconductors were originally observed [12,13] and identified [14] in disordered quantum wires, where they result from a break-up of the narrow channel into a few segments separated by tunnel tarriers. A theory for the Coulomb-blockade oscillations in the quantum regime $k_{\mathrm{B}} T \leqslant \Delta E$ has been developed re- 


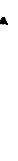


cently $[15,16]$. Here we present new experimental results on the Coulomb-blockade oscillations in a quantum dot. In contrast to disordered quantum wires, the tunnel barriers in a quantum dot are created artificially, allowing for more experimental control. One of our findings is that by applying a strong magnetic field the amplitude of the oscillations can be enhanced up to $e^{2} / h$.

The sample that we have used for our experiments (fig. 1(a)) is based on a GaAs-AlGaAs heterostructure containing a $2 \mathrm{DEG}$ with a sheet electron density of $2 \times 10^{11} \mathrm{~cm}^{-2}$ and a mobility of $2 \times 10^{6} \mathrm{~cm}^{2} / \mathrm{Vs}$, at low temperatures. The quantum dot is defined by means of electrostatic depletion of the $2 \mathrm{DEG}$ using three pairs of gates, as shown schematically in fig. 1(b). Two adjustable tunnel barriers are formed by opposite quantum point contacts (of $0.3 \mu \mathrm{m}$ lithographic width and $0.7 \mu \mathrm{m}$ separation) defined by gates $(\mathrm{B}, \mathrm{C})$ and $(\mathrm{E}, \mathrm{F})$. The number of electrons confined in the quantum dot can be controlled by two additional gates (A, D) $(0.9 \mu \mathrm{m}$ apart). The gaps between the point-contact gates and the control gates are approximately $0.2 \mu \mathrm{m}$ wide, so that the quantum dot is formed before the point contacts are pinched off. The measurements of the conductance were carried out using an $\mathrm{AC}$ lock-in technique, with the sample mounted in the mixing chamber of a dilution refrigerator. The temperature was approximately $100 \mathrm{mK}$, and the source-drain voltage was $10 \mu \mathrm{V}$. The diagonal measurement configuration shown in fig. 1(b) yields an effective two-terminal conductance, even if a magnetic field is applied [4].

In fig. 2 we show experimental results of the Coulomb-blockade oscillations as a function of the voltage $V_{\text {gate }}$ applied to the control gates (A, $\mathrm{D})$, for six values of the magnetic field between $B=0$ and $3.75 \mathrm{~T}$. The ficld was oriented perpendicular to the 2DEG. The point contacts were adjusted to the tunneling regime $\left(G<2 e^{2} / h\right.$, at

Fig 1 (d) Scanning-electron micrograph of the quantum dot (b) Schematic top-view of the quantum dot Gates $(B, C)$ and $(E, F)$ definc tunnel barriers of adjustable transparency Gates $(A, D)$ control the number of electrons confined in the quantum dot. The current contacts are labelled by $I_{1}, I_{2}$, the voltage contacts by $V_{1}, V_{2}$ (c) Equivalent circuit of the quantum dot and the control gates $(A, D)$

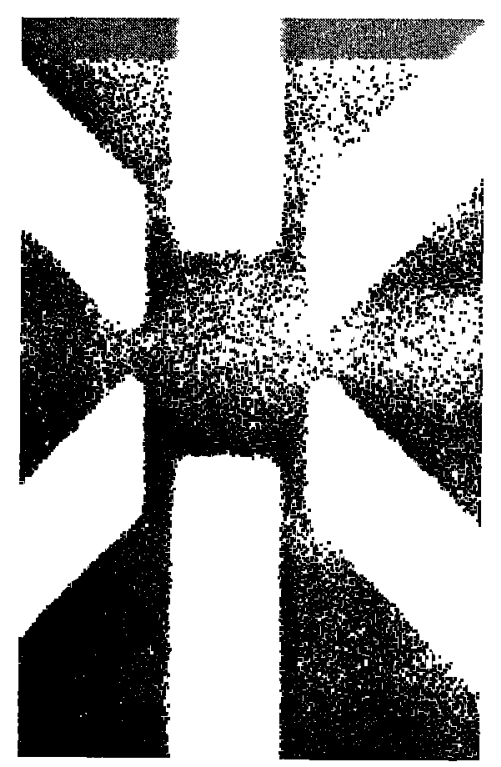

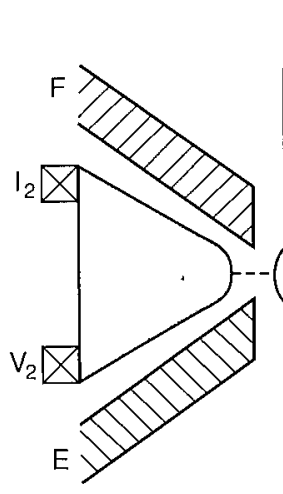

(b)
A

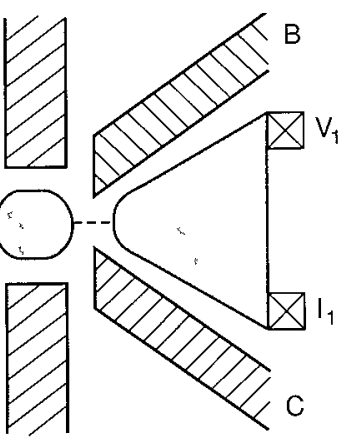

$\mathrm{D}$

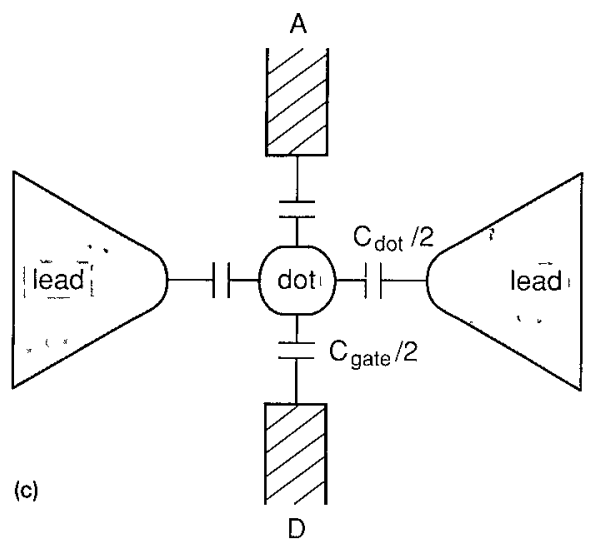




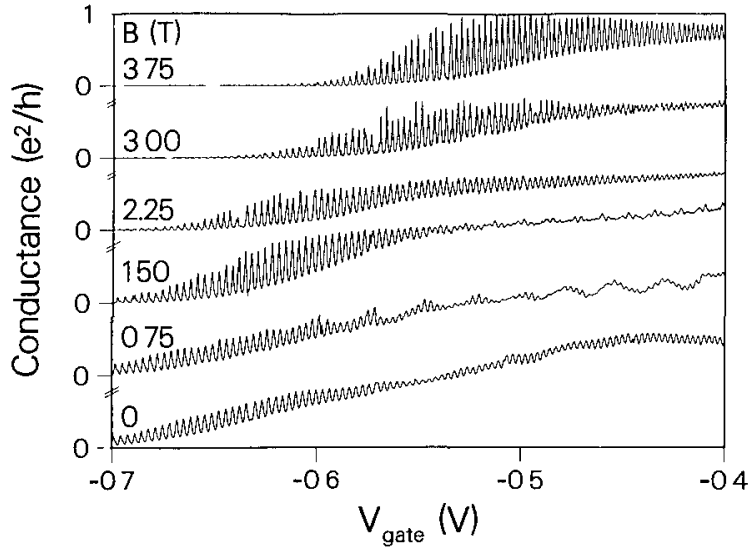

Fig. 2. Equivalent two-terminal conductance of the quantum dot versus voltage on the control gates (A, D) (applied relative to one of the current contacts), for six values of a perpendicular magnetic field. The voltages on the pointcontact gates $(B, C)$ and $(E, F)$ were kept fixed, at a value such that the zcro-field conductance of the individual point contacts was below $2 e^{2} / h$.

$B=0$ ). No oscillations were observed if either or both point contacts were set to a conductance above $2 e^{2} / h$ (at $B=0$ ), in agreement with ref. [9]. All traces show a large number of oscillations which are nearly periodic in the gate voltage. The period of the oscillations increases slowly as the gate voltage is made more negative, presumably because the size of the quantum dot is decreased (thereby decreasing the mutual capacitance of quantum dot and control gates). Near $V_{\text {gate }}=-0.4 \mathrm{~V}$ the period of the $B=0$ trace is $\Delta V_{\text {gatc }} \approx 2.8 \mathrm{mV}$, while near $V_{\text {gatc }}=-0.7 \mathrm{~V}$ it is approximately $3.4 \mathrm{mV}$.

A magnetic field has almost no effect on the period (less than a few percent for $B<4 \mathrm{~T}$ ), and no spin splitting of the conductance peaks is observed. An additional slow modulation with a period of approximately $26 \mathrm{mV}$ is observed for $V_{\text {gate }} \geqslant-0.6 \mathrm{~V}$ at $B=0.75 \mathrm{~T}$. At $B=3.75 \mathrm{~T}$ an apparent doublet structure is seen in a small voltage interval. These secondary effects, which are not understood, are similar to those observed in disordered quantum wires [13]. The gate voltage at which the oscillations disappear, and the conductance vanishes, increases monotonically with increasing magnetic field. We attribute this to a magnetic depopulation of the quantum dot.
The peak-to-valley amplitude of the oscillations does not exceed $e^{2} / h$, and typically exhibits a maximum for intermediate gate-voltages. They are superimposed on a background conductance, which increases slowly with gate voltage. We attribute the $V_{\mathrm{gdte}}$-dependence of the amplitude of the oscillations to the effect of the control gates $(A, D)$ on the transparency of the potential barriers in the boundary of the quantum dot. At more negative gate voltages, the tunnel barriers in the point contacts become less transparent, reducing the peak height. At less negative gate voltages, the potential barriers in the gaps between the control and point-contact gates become more transparent, increasing quantum fluctuations in the charge of the quantum dot. This leads to an increased background conductance, and to conductance maxima exceeding $e^{2} / h$ in the zero-field trace. It is not yet clear whether this is due to virtual tunneling processes [17, 18], known to be important in metals if the conductance of the individual barriers is of the order of $e^{2} / h$, or to a cross-over from tunneling to ballistic transport through the point contacts. A dynamical treatment is required in our case of low tunnel barriers, since the field across the barrier changes during the tunnel process [19]. Similar dynamic polarization effects are known to play a role in large-area semiconductor tunnel junctions, where they are related to an image-force lowering of the barrier height.

The amplitude of the oscillations depends nonmonotonically on $B$. In the presence of a magnetic field the oscillations are quite spectacular, of amplitude approaching $e^{2} / h$ (at $B=3.75 \mathrm{~T}$ and $V_{\text {gitc }} \approx-0.52 \mathrm{~V}$, see fig. 2). Whereas the amplitude of the oscillations is enhanced, the background conductance is reduced, in such a way that $G<e^{2} / h$ in the entire conductance trace.

Let us now estimate the relevant energies in the problem. The area of the quantum dot in the relevant gate-voltage regime is estimated at $A=$ $0.4 \times 0.6 \mathrm{\mu m}^{2}$ (assuming that the lateral depletion length equals half the lithographical width of the point contacts). In the absence of a magnetic field, this implies $\Delta E \approx 2 / \rho_{2 \mathrm{D}} A \approx 30 \mu \mathrm{eV}$, where $\rho_{2 \mathrm{D}}$ is the two-dimensional density of states (for one spin direction). Our experimental observa- 
tion of oscillations up to $T \approx 09 \mathrm{~K}$ implies an activation energy of the conductance minima of about $4 k_{\mathrm{B}} T \approx 03 \mathrm{meV}$ Clearly, this excludes an inteipretation of the oscillations in terms of resonant tunneling of non-interacting electrons This conclusion is supported further by the absence of spin splitting in a magnetic field The charging energy $e^{2} / C$ can be estimated from the self-capacitance of a flat circular disk of diameter $d, \quad C=4 \varepsilon_{0} \varepsilon_{\mathrm{r}} d \approx 3 \times 10{ }^{16} \mathrm{~F} \quad\left(\varepsilon_{\mathrm{r}}=13\right.$ and $d=$ $06 \mu \mathrm{m})$ Our estımate $e^{2} / C \approx 05 \mathrm{meV}$ explains the large activation energy of the conductance minıma in the experiment, as we now discuss

As shown in ref [20], the condition for a conductance peak due to resonant tunneling in the presence of charging effects is (at $T=0$ )

$$
E_{N}^{\vdots} \equiv E_{N}+\left(N-\frac{1}{2}\right) \frac{e^{2}}{C}=e \phi_{\text {ext }}+E_{\Gamma},
$$

where $N$ is the number of electrons in the quantum dot, $E_{\mathrm{F}}$ the Fermi energy of the reservoirs, and $\phi_{\mathrm{cxt}}=\phi_{\text {donor }}+\alpha \phi_{\mathrm{g} \text { itc }}$ that part of the potential difference between the quantum dot and the reservoirs which is due to the fixed charge on the 1onized donors and a variable charge on the gate ( $\alpha$ is a rational function of the capacitance matrix elements of the system) The energy $E_{N}$ of the $N$ th level is measured relative to the conduction band bottom in the quantum dot The effective energy level separation $\Delta E^{*}=\Delta E+e^{2} / C$ for transport is enhanced and regulated by the charging energy The activation energy of the minima is ${ }_{2}^{1} \Delta E^{4}$ [15] Since in our experiment $e^{2} / C \gg \Delta E$, this explains our observation of periodic oscillations in the conductance at temperatures much above those expected for resonant tunneling of non-interacting electrons In addition, the spin degeneracy of the effective energy levels in the quantum dot is lifted by the charging energy, explainıng the absence of spin splitting of the oscillations, even in a strong magnetic field

Whereas the temperature dependence of the Coulomb-blockade oscillations is determined by the total capacitance $C$ of the quantum dot, the period is determined by the smaller mutual capacitance $C_{\text {tite }}$ of the quantum dot and the control gates $(\mathrm{A}, \mathrm{D})$ We represent the system of quantum dot, control gates, and 2DEG leads by the equivalent circuit of fig $1(\mathrm{c})$ (the mutual capacitance between the quantum dot and the point-contact gates is absorbed in $C_{\text {dot }}$ ) Using simple electrostatics, one finds $\alpha=C_{\mathrm{g} \text { tcc }} / C$, where $C \equiv C_{\text {titc }}+C_{\text {dot }}$ Since the control gates affect primarily the quantum dot and not the reservorrs, $E_{\mathrm{F}}$ can be assumed to be constant Equation (1) then implies a perıod*

$\Delta \phi_{\text {gite }}=\frac{e}{C_{\text {gul }}}\left[1+\frac{\Delta E}{e^{3} / C}\right]$

In the case $\Delta E \ll e^{\gamma} / C$ the period is constant, even if the level separation is non-uniform

In the experiment, the estımated level separatıon $\Delta E$ between spin-degenerate levels is only $5 \%$ of $e^{2} / C$ From the period of the oscillations we find $C_{\text {sit }} \approx 5 \times 10^{-17} \mathrm{~F}$, an order of magnitude smaller than $C$ The increase of the period for $V_{\text {sitc }}$ approdching $-07 \mathrm{~V}$ thus suggests a decreased dot-gate capacitance $C_{\text {gate, }}$, which is not unreasonable in view of the lateral depletion technique used The period is found to be almost independent of the magnetic field, indicating that $C_{\text {gitc }}$ does not depend strongly on the magnetic field The effect of the magnetic field on the energy levels in the quantum dot may be large, but this does not strongly affect the period of the oscillations in our experiment, since $\Delta E \ll e^{\hat{T}} / C$

In conclusion, we have observed periodic oscillations in the conductance of a quantum dot as a function of gate voltage From the fact that these oscillations are observed at relatively high temperatures, compared to the energy level separation in the quantum dot, we conclude that they are Coulomb-blockade oscillations, rather than oscillations due to resonant tunneling The period of the oscillations is in qualitative agreement with theoretical estimates The effect of a magnetic field on the amplitude of the oscillations and the background conductance remains to be understood

\footnotetext{
* We note that the controlled gate voltage $V_{\mathrm{g} \text { ic }}$ is the electro chemical potentral difference between gates and leads rather than the electrostatic potential difference $\phi_{\text {a ate }}$ How ever the change in Ferm energy in the (metal) gates is negligible within one period and therefore $\Delta V_{\mathrm{g} \text { ic }}=\Delta \phi_{\mathrm{k}-11}$
} 


\section{Acknowledgements}

We thank M.A A. Mabesoone and C E. Timmering for technical support, B.W. Alphenaar, S. Colak and LW Molenkamp for stimulatıng discussions, and MFH Schuurmans and J.H. Wolter (Einhoven University of Technology) for their support and interest. This research was partly funded by the ESPRIT basic research action project 3133

\section{References}

[1] B J van Wees, H van Houten, CW J Becnakker, J G Williamson, L P Kouwenhoven, D van der Marel and C T Foxon, Phys Rev Lett 60 (1988) 848

[2] D A Wharam, T J Thornton, R Newbury, M Pepper $H$ Ahmed, J E F Frost, D G Hasko, D C Peacock, D A Ritchie and G A C Jones, J Phys C 21 (1988) L209

[3] $\mathrm{H}$ van Houten and CW J Beenakker, in Analogies in Optics and Microelectronics, eds W van Haeringen and D Lenstra (Kluwer, Dordrecht, 1990)

E A Montre, E C Cosman, GW 't Hooft, M B van der Mark and CW J Beenakker, Nature 350 (1991) 594

[4] For a review sec CW J Beenakker and $H$ van Houten, Solid State Phys 44 (1991) 1

[5] C G Smith, M Pepper, H Ahmed, J E F Frost, D G Hasko, D C Peacock, D A Ritchie and G A C Jones, J Phys C 21 (1988) L893

[6] B J van Wees, L P Kouwenhoven. C J P M Harmans, J G Willamson, C E Timmering, M E I Brockaart, C T Foxon and J I Harris, Phys Rev Lett 62 (1989) 2523
[7] U Meirav, M A Kelstner and S J Wind, Phys Rev Lett 65 (1990) 771

81 P L McEuen, E B Foxman, U Meırav, M A Kastnet, Y Merr, N S Wingreen and SJ Wind, Phys Rev Lett 66 (1991) 1926

[9] L P Kouwenhoven, N C van der Vaart, A T Johnson, C J P M Harmans, J G Willamson, A A M Starıng and $\mathrm{CT}$ Foxon, Festkorperprobleme, Advances in Solid State Physics, Vol 31, to be published

[10] For a review see K K Likharev, IBM J Res Dev 32 (1988) 144

[11] For a review see $\mathrm{H}$ van Houten, CWJ Beenakker and A A M Staring, in Single Charge Tunnelıng, eds H Grabert and M H Devoret (Plenum, New York, 1991)

[12] J H F Scott-Thomas, S B Field, M A Kastner, H I Ficld and D A Antoniadis, Phys Rev Lett 62 (1989) 583

[13] A A M Starıng, H van Houten, CW J Beenakker and C T Foxon, in High Magnetic Fields in Semiconductor Physics III, ed G Landwehr (Springer, Berlın, 1991)

[14] $\mathrm{H}$ van Houten and C W J Bcenakker, Phys Rev Lett 63 (1989) 1893

[15] CW J Beenakker, Phys Rev B 44 (1991) 1646

[16] Y Meir, N S Wingreen and PA Lee, Phys Rev Lett 66 (1991) 3048

[17] DV Averm and A A Odintsov, Phys Lett A 140 (1989) 251

DV Averin and YuV Nazarov Phys Rev Lett 65 (1990) 2446

[18] L I Glazman and K A Matvecv, Pis'ma Zh Eksp Teor F1Z 51 (1990) 425 (JETP Lett 51 (1990) 484)

[19] Yu V Nazarov, Solid State Commun 75 (1990) 669 A N Korotkov and YuV Nazarov, Physica B 173 (1991) 217

[20] CW J Beenakker, H van Houten and A A M Staring, Phys Rev B 44 (1991) 1657 